

\section{DISCLAIMER}

This report was prepared as an account of work sponsored by an agency of the United States Government. Neither the United States Government nor any agency Thereof, nor any of their employees, makes any warranty, express or implied, or assumes any legal liability or responsibility for the accuracy, completeness, or usefulness of any information, apparatus, product, or process disclosed, or represents that its use would not infringe privately owned rights. Reference herein to any specific commercial product, process, or service by trade name, trademark, manufacturer, or otherwise does not necessarily constitute or imply its endorsement, recommendation, or favoring by the United States Government or any agency thereof. The views and opinions of authors expressed herein do not necessarily state or reflect those of the United States Government or any agency thereof. 


\section{DISCLAIMER}

Portions of this document may be illegible in electronic image products. Images are produced from the best available original document. 
An Affirmative Action/Equal Opportunity Employer

\section{DISCLAIMER}

This report was prepared as an account of work sponsored by an agency of the United States Government. Neither the United States Government nor any agency thereof, nor any of their employees, makes any warranty, express or implied, or assumes any legal liability or responsibility for the accuracy, completeness, or usefulness of any information, apparatus, product, or process disclosed, or represents that its use would not infringe privately owned rights. References herein to any specific commercial product, process, or service by trade name, trademark, manufacturer, or otherwise, does not necessarily constitute or imply its endorsement, recommendation, or favoring by the United States Government or any agency thereof. The views and opinions of authors expressed herein do not necessarily state or reflect those of the United States Government or any agency thereof. 
UC-32

Issued: February 1982

\section{Accurate}

\section{Monotonicity-Preserving \\ Cubic Interpolation}

James $M$. Hyman

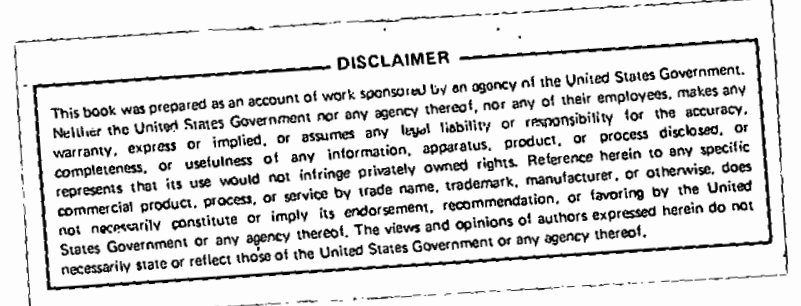

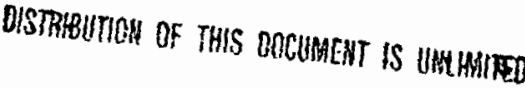

MGW

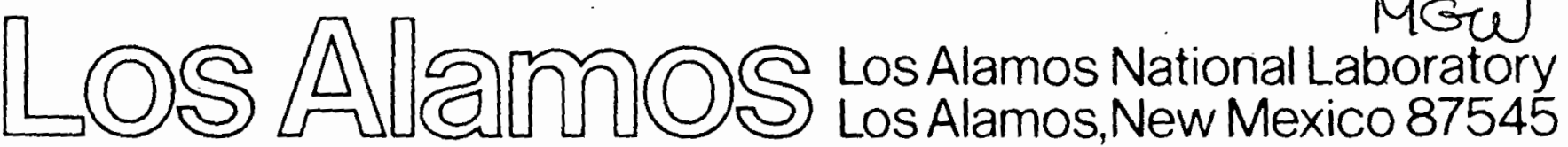




\section{PAGES ii to iv WERE INTENTIONALLY LEFT BLANK}


CONTENTS

ABSTRACT . . . . . . . . . . . . . . . . . . . . . . . 1

I. INTRODUCTION . . . . . . . . . . . . . . . . . . 2

II. CUBIC HERMITE INTERPOLATION . . . . . . . . . . . . . . . . . . 3

A. The Interpolation Formula .. . . . . . . . . . . . . . 4

B. Monotonicity.................... . 5

III. DERIVATIVE APPROXIMATION . . . . . . . . . . . . . . . . . 8

A. Local Methods . . . . . . . . . . . . . . . . . . . . . 9

B. Nonlocal Methods..................... . . 11

C. Boundaries . . . . . . . . . . . . . . . . . 12

IV. NUMERICAL EXAMPLES . . . . . . . . . . . . . . . . . . . 13

A. Monotone Example . . . . . . . . . . . . . . . . . . . 14

B. Nonmonotone Example . . . . . . . . . . . . . . . . . . . 14

V. SUMMARY AND CONCLUSIONS . . . . . . . . . . . . . . . . . . 17

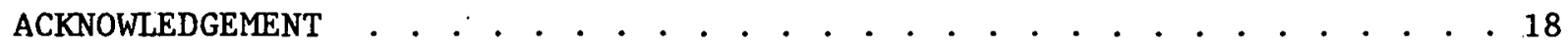

REFERENCES . . . . . . . . . . . . . . . . . . . . . 18

$\mathrm{v}$ 


\title{
ACCURATE MONOTONICITY-PRESERVING CUBIC INTERPOLATION
}

by

James M. Hyman

\begin{abstract}
A simple and effective algorithm to construct a monotonicity-preserving cubic Hermite interpolant for data with rapid variations is presented. Constraining the derivatives of the interpolant according to geometric considerations makes the interpolant consistent with local monotonicity properties of the data. Numerical examples are given that compare the quality and accuracy of the proposed interpolation method with other standard interpolants.
\end{abstract}




\section{INTRODUCTION}

Piecewise polynomial interpolation is used to deduce probable values for an implied function defined at a discrete set of points. For an accurate interpolation, we must carefully retain crucial properties of the data (such as monotonicity or convexity), and we must not introduce details or artifacts that cannot be ascertained from the data. This requires that the interpolant be designed according to both geometric and algebraic considerations.

The geometric qualities of an interpolant are based on how well the interpolated curve reflects the intrinsic shape inferred by the data points. A good geometric interpolant will produce a curve similar to the visually pleasing one of a draftsman. Although some mathematical properties, such as those that preserve monotonicity and convexity, can describe the goodness of fit in a geometric sense, choosing the better curve often is a heuristic decision based on human judgment rather than on firm mathematical theory.

A good geometric interpolant is most important when the data arise from a physical experiment and an underlying mathematical structure does not exist. For these data sets, geometric considerations, such as preventing spurious behavior near rapid changes in the data, may be more important than the method's asymptotic accuracy. In fact, maintaining monotonicity or convexity in the interpolation process may be necessary to represent physical reality. For example, if the data come from an equation-of-statc table for density vs pressure, then a nonmonotone interpolant will have a negative derivative and will imply an imaginary sound speed for the material. ${ }^{1}$ This error can destroy the accuracy of any calculation based on the interpolated data.

The functional or algebraic properties of a good interpolant in classic approximation theory are defined more precisely. ${ }^{2}$ They include the order of accuracy as the mesh spacing becomes arbitrarily small, continuity or 
smoothness of some derivative of the interpolant, and invariance or linearity properties such as

$$
P(\alpha f+g)=\alpha P f+P g,
$$

where $P$ is the interpolation operator, $\alpha$ is a scalar, and $f$ and $g$ are functions. Note that none of these properties guarantee a good geometric interpolant.

Another consideration is a method's practicality, as evidenced by its simplicity, efficiency, and storage requirements. In this report, we restrict our analysis to local piecewise polynomial interpolants that, when compared to other methods, rate high in these three categories.

We have developed and tested a practical algorithm that is excellent both geometrically and algebraically and is highly accurate when monotonicity properties of the interpolant do not intervene. Loss of accuracy in favor of shape preservation occurs only at isolated points where the grid is rough compared to the solution variation.

First, we briefly describe the piecewise polynomial cubic Hermite interpolant and the restrictions sufficient to guarantee monotonicity. We then describe some possible algorithms that compute the derivatives needed by the interpolant at the mesh points, and we give numerical examples that compare our method to similar methods.

\section{CURIC HERMITE INTERPOLATION}

Let the mesh $\left\{\mathrm{x}_{i}\right\}_{i=1}^{\mathrm{n}}$ be a partition $\mathrm{x}_{1}<\mathrm{x}_{2}<\cdots<\mathrm{x}_{\mathrm{n}}$ of the interval $\left[x_{1}, x_{n}\right]$ and let $\left\{f_{i}\right\}, f_{i}=f\left(x_{i}\right)$ be the corresponding data points. The local mesh spacing is $\Lambda x_{i+\frac{1}{2}}=x_{i+1}-k_{1}$, and the slope of the piecowise linear interpolant between the data points is $\mathrm{S}_{i+\frac{1}{2}}=\Delta \mathrm{f}_{i+\frac{1}{2}} / \Delta \mathrm{x}_{i+\frac{1}{2}} \cdot$ The data are 
locally monotone at $x_{i}$ if $S_{i+\frac{1}{2}} S_{i-\frac{1}{2}}>0$. The interpolant is piecewise monotone if $(P f)(x)$ is monotone between $f_{i}$ and $f_{i+1}$ for $x$ between $x_{i}$ and $x_{i+1}$. The interpolant $P f$ is class $C^{k}$ if $(P f)(x)$ is continuous and has continuous derivatives for all orders less than or equal to $k$.

\section{A. The Interpolation Formula}

Given the data points $\left\{f_{i}\right\}$, a numerical approximation of the slope $\dot{f}_{i}$ at $\mathbf{x}_{i}$ is calculated for $1 \leq \mathrm{i} \leq \mathrm{n}$. The cubic Hermite interpolant then is defined for $1 \leq i<n$ as

$$
P(x)=c_{1}+\left(x-x_{i}\right) c_{2}+\left(x-x_{i}\right)^{2} c_{3}+\left(x-x_{i}\right)^{3} c_{4},
$$

where

$$
\begin{aligned}
& x_{i} \leq x \leq x_{i+1}, \\
& c_{1}=f_{i}, \\
& c_{2}=\dot{f}_{1}, \\
& c_{3}=\left(3 S_{i+\frac{1}{2}}-\dot{f}_{i+1}-2 \dot{f}_{i}\right) /\left(\Delta x_{i+\frac{1}{2}}\right),
\end{aligned}
$$

and

$$
c_{4}=-\left(2 s_{i+\frac{1}{2}}-\dot{\mathbf{f}}_{i+1}-\dot{\mathrm{f}}_{i}\right) /\left(\Delta \mathrm{x}_{i+\frac{1}{2}}^{2}\right)
$$

The interpolant Eq. (2.1) has a continuous first derivative, $p(x) \varepsilon C^{1}$, and possibly, but not necessarily, a continuous second derivative. The continuity of the second derivative and the order of accuracy depend on how $\left\{\dot{\mathrm{f}}_{i}\right\}$ are calculated.

Note that once $\left\{\dot{\mathrm{f}}_{i}\right\}$ are given, Eq. (2.1) becomes a local interpolation formula. By changing the value of $f_{i}$ or $\dot{\mathrm{f}}_{i}$ at a data point, the interpolant 
changes only in the region $\left[x_{i-1}, x_{i+1}\right]$. If the calculation of $\dot{f}$ also is local, only nearby data points need be available when interpolating between $x_{i}$ and $x_{i+1}$. This localness is an important quality when storage requirements are critical as is the case for very large data sets or multidimensional interpolation.

Localness of the interpolant also is desirable when data are being readjusted a few points at a time. This occurs in interactive graphics routines to avoid recalculating the interpolation function at all data points.

The numerical approximation of $\left\{\dot{f}_{i}\right\}$, which makes Eq. (2.1) a $C^{2}$ interpolant (for example, the complete spline interpolant ${ }^{2}$ ), is not local. Thus, to gain total localness for Eq. (2.1), we must sacrifice global continuity in the second derivative.

B. Monotonicity

Even when $\left\{\dot{f}_{i}\right\}$ are defined accurately, additional constraints may be necessary because Eq. (2.1) may fail to produce an acceptable interpolant in the geometric sense for certain data sets. A simple generalization of what was recognized by de Boor and $S_{w a r t z}{ }^{3}$ is that if the data are locally monotonically increasing at $x_{i}$ and if

$$
0 \leq \dot{f}_{j} \leq 3 \min \left(s_{j-\frac{1}{2}}, s_{j+\frac{1}{2}}\right)
$$

for $j=i$ or $i+1$, then the resulting interpolant is monotone in $\left[x_{i}, x_{i+1}\right]$. Fritsch and Carlson ${ }^{4}$ independently found an extension of this criterion giving a necessary and sufficient condition for (2.1) to be monotone. The de BoorSwartz criterion is a square inscribed within the Fritsch-Carlson monotonicity region. 
Note that if $\left\{\dot{\mathrm{f}}_{i}\right\}$ are calculated to make the resulting interpolant $\mathrm{C}^{2}$, then Eq. (2.2) may not be satisfied. That is, there are monotone data sets for which there is no $c^{2}$ piecewise-cubic Hermite interpolant.

When the data are locally monotone, we restrict $\left\{\dot{\mathrm{f}}_{i}\right\}$ to the de BoorSwartz piecewise monotonicity range of Eq. (2.2) as follows. After calculating an accurate approximation of $\dot{\mathbf{f}}_{i}$ (for instance, finite differences or by the complete spline formula), we project it to the allowed monotonicity region according to

$$
\dot{f}_{i} \leftarrow \begin{cases}\min \left[\max \left(0, \dot{f}_{i}\right), 3 s_{\min }^{i}\right] \text { if } 0<s_{\min }^{i} \\ \max \left[\min \left(0, \dot{\mathrm{f}}_{i}\right), 3 \mathrm{~s}_{\max }^{i}\right] \text { if } 0>\mathrm{s}_{\max }^{i} \\ 0 & \text { if } 0 \geq s_{i-\frac{1}{2}} s_{i+\frac{1}{2}},\end{cases}
$$

where

$$
S_{\min }^{i}=\min \left(S_{i-\frac{1}{2}}, S_{i+\frac{1}{2}}\right)
$$

and

$$
S_{\max }^{i}=\max \left(S_{i-\frac{1}{2}}, S_{i+\frac{1}{2}}\right)
$$

Near the boundary, the de Boor-Swartz constraint can be used by letting $S_{-\frac{1}{2}}=S_{\frac{3}{2}}$ and $S_{n+\frac{1}{2}}=S_{n-\frac{1}{2}}$.

When a $\dot{f}_{i}$ associated with a complete spline interpolant falls outside the range of Eq. (2.2), as it inevitably will when the variation between the data points is large, resetting $\dot{f}_{i}$ according to Eq. (2.3) will cause the second derivative of the interpolant to jump where $\dot{f}_{i}$ was reset and at the two nearest mesh points.

If the underlying function is strictly monotone and sufficiently smooth and $\dot{\mathbf{f}}_{i}$ is an accurate approximation of the derivative at $x_{i}$, then as the mesh 
is refined, Eq. (2.2) will be satisfied in the limit because

$$
\begin{aligned}
\left.\frac{\mathrm{df}}{\mathrm{dx}}\right|_{x=x_{i}} & \dot{\mathrm{f}}_{i}+o\left(\Delta \mathrm{x}^{?}\right) \\
& =\mathrm{s}_{i+\frac{1}{2}}+0(\Delta \mathrm{x}) \\
& =\mathrm{s}_{i-\frac{1}{2}}+0(\Delta \mathrm{x}) .
\end{aligned}
$$

Thus, the interpolant is restricted by geometric considerations only when the mesh is coarse and the asymptotic accuracy in $\dot{\mathrm{f}}$ is meaningless. When the mesh accurately resolves the function implied by the data, the accuracy in $\dot{\mathfrak{f}}$ is retained because Eq. (2.2) will be satisfied.

When the data are not locally monotone, the interpolant also must have an extrema. Retaining piecewise monotonicity would require that $\dot{f}_{i}=0$ and would "clip" the interpolant by forcing inter-interval monotonicity on nonmonotone data. However, the piecewise monotonicity constraint can be relaxed in the interval pair next to the extrema to produce (in the author's opinion) a more visually pleasing curve. But if a new constraint is imposed at extrema, the change in decision algorithms must still produce a stable interpolant. That is, a small change in the data should not create a large change in the interpolant. If we remove all constraints on the interpolant near locally nonmonotone data while retaining Eq. (2.3) elsewhere, the resulting interpolant will be unstable.

We chose to extend Eq. (2.2) by requiring that $\dot{f}_{i}$ have the same sign as either $\mathrm{S}_{i-\frac{1}{2}}$ or $\mathrm{S}_{i+\frac{1}{2}}$, whichever is larger, and that

$$
\left|\dot{f}_{i}\right| \leq 3 \min \left(\left|s_{i-\frac{1}{2}}\right|,\left|S_{i+\frac{1}{2}}\right|\right) .
$$


The constraining function extending Eq. (2.3) is

$$
\dot{f}_{i} \leftarrow\left\{\begin{array}{l}
\min \left[\max \left(0, \dot{f}_{i}\right), 3 \min \left(\left|s_{i-\frac{1}{2}}\right|,\left|s_{i+\frac{1}{2}}\right|\right)\right], \sigma>0 \\
\max \left[\min \left(0, \dot{f}_{i}\right),-3 \min \left(\left|s_{i-\frac{1}{2}}\right|,\left|s_{i+\frac{1}{2}}\right|\right)\right], \sigma<0,
\end{array}\right.
$$

where if $\left|S_{i-\frac{1}{2}}\right|>\left|S_{i+\frac{1}{2}}\right|$, then $\sigma=\operatorname{sign}\left(S_{i-\frac{1}{2}}\right) ;$ otherwise $\sigma=\operatorname{sign}\left(S_{i+\frac{1}{2}}\right)$.

The sign function $\operatorname{sign}(S)=1$ if $S \geq 0$ and -1 otherwise.

Often the monotonicity of the interpolant's derivative is an important quality that can be incorporated into the interpolant; if the data are convex, a good geometric interpolant should preserve this convexity. However, a $\mathrm{C}^{1}$ convexity-preserving cubic Hermite interpolant does not exist for all data sets. $^{5}$ For example, a $c^{1}$ convex interpolant does not exist for $f=x+|x|$ when $x=0$ is a data point. If the $c^{1}$ constraint is dropped, restrictions similar to Eq. (2.6) can be incorporated in Eq. (2.1) to preserve convexity. ${ }^{6}$

A simple, necessary, but not sufficient, and often effective convexitypreserving constraint involves limiting the $\left\{\dot{f}_{i}\right\}$ so

$$
\min \left(S_{i-\frac{1}{2}}, s_{i+\frac{1}{2}}\right) \leq \dot{y}_{i} \leq \max \left(S_{i-\frac{1}{2}}, s_{i+\frac{1}{2}}\right)
$$

by using

$$
\dot{y}_{i} \leftarrow \max \left\{\min \left[\dot{y}_{i}, \max \left(s_{i-\frac{1}{2}}, s_{i+\frac{1}{2}}\right)\right], \min \left(s_{i-\frac{1}{2}}, s_{i+\frac{1}{2}}\right)\right\}
$$

\section{DERIVATIVE APPROXIMATION}

The order of accuracy of (2.1) can be, at best, one order higher than the order of accuracy of $\dot{f}_{i}$. Therefore, it is prudent to calculate $\dot{f}_{i}$ accurately whenever possible. The difference approximations can be divided into two classes: local and nonlocal. The local schemes use only $f$ values near $x_{i}$ to calculate $\dot{\mathrm{f}}_{i}$. The nonlocal schemes use all $\left\{\mathrm{f}_{i}\right\}$ values and obtain $\left\{\dot{f}_{i}\right\}$ by solving a linear system of equations. 


\section{A. Local Methods}

De Boor and Swartz have shown that there are no linear algorithms yielding derivative approximations above first order that also automatically satisfy (2.2). There are, however, many nonlinear formulas that do. The Butland $^{7}$ algorithm, for example, yields $\left\{\dot{f}_{i}\right\}$, which automatically satisfies (2.2) and is second order on a uniform grid. The Fritsch-Butland ${ }^{8}$ algorithm listed in Table $\mathrm{I}$ is a slight modification of this formula.

The parabolic interpolation method in Table $I$ is linear, and the resulting $\left\{\dot{f}_{i}\right\}$ do not automatically satisfy (2.2). This formula can be multiplied by a nonlinear factor with magnitude $1+0\left(\Delta x^{2}\right)$ to give the monotonicity-preserving formula

$$
\dot{f}_{i}=\frac{(2+\theta) s_{i+\frac{1}{2}} s_{i-\frac{1}{2}}}{s_{i+\frac{1}{2}}^{2}+s_{i-\frac{1}{2}}^{2}+\theta s_{i+\frac{1}{2}} s_{i-\frac{1}{2}}} \frac{\Delta x_{i-\frac{1}{2}} s_{i+\frac{1}{2}}+\Delta x_{i+\frac{1}{2}} s_{i-\frac{1}{2}}}{\Delta x_{i-\frac{1}{2}}+\Delta x_{i-\frac{1}{2}}}
$$

This formula is second order for monotone data when

$$
-2<\theta \leq 1+3 \min \left(\Delta x_{i+\frac{1}{2}} / \Delta x_{i-\frac{1}{2}}, \Delta x_{i-\frac{1}{2}} / \Delta x_{i+\frac{1}{2}}\right)
$$

The tests for the resulting interpolant with $\theta=1$ are not included in this report but are similar to and only slightly less accurate than the monotonicity-constrained parabolic interpolant.

The fourth-order finite difference method ${ }^{10}$ in Table $I$ is based on first mapping $\left\{x_{i}\right\}$ to a standard equally spaced reference grid $\left\{r_{i}\right\}$ and then approximating each term in the identity

$$
\frac{d f}{d x}=\frac{d f}{d r}\left(\frac{d x}{d r}\right)^{-1}
$$

with centered fourth-order finite differences for an equally spaced grid. If 
LOCAL FORMULAS FOR $\dot{\mathrm{f}}_{i}$ AND THEIR ORDER OF ACCURACY FOR SMOOTH FUNCTIONS AND MESH VARIATIONS

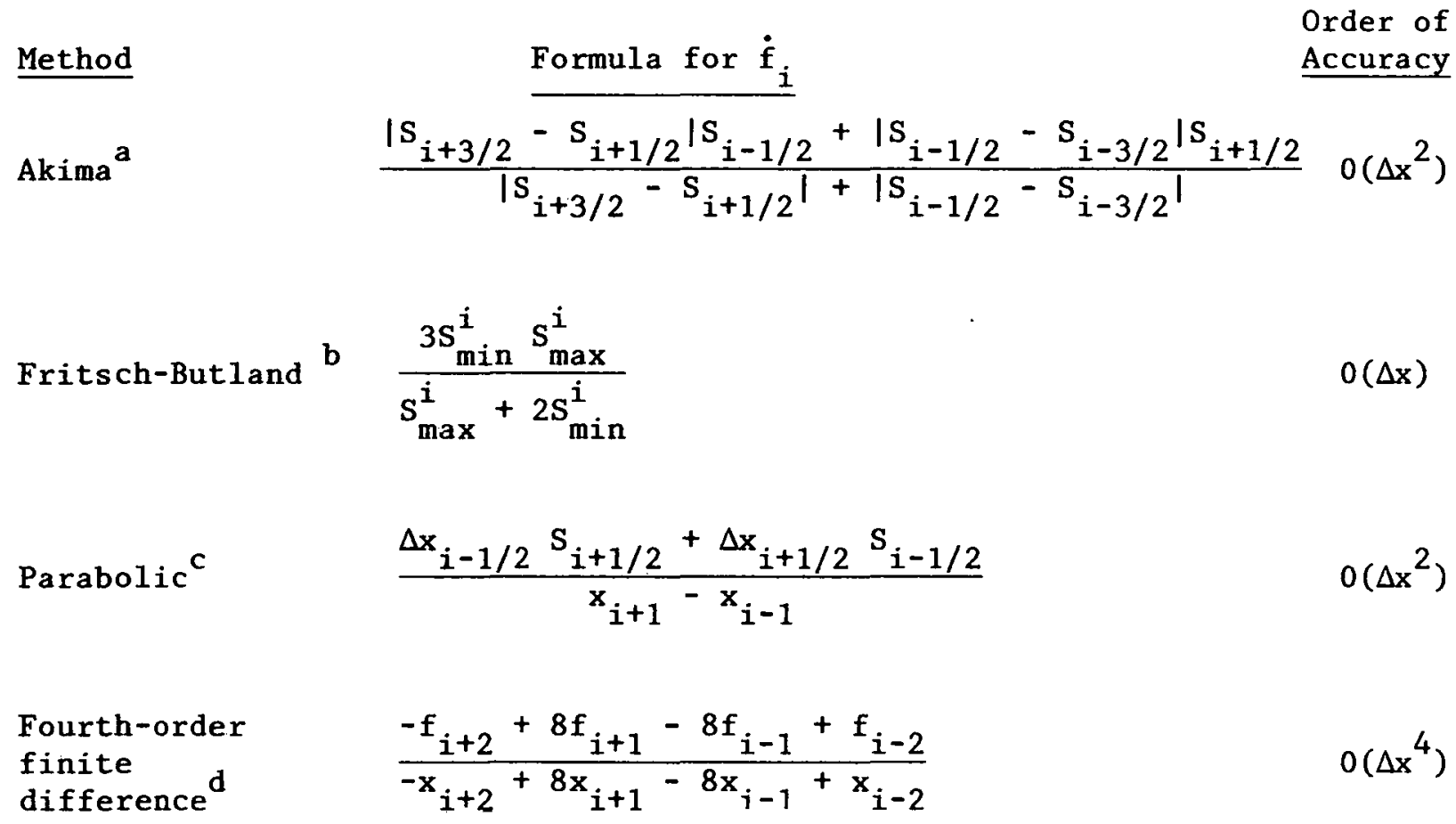

${ }^{a}$ See Ref. 2.
${ }^{b}$ See Ref. 9.
${ }^{c}$ See Ref. 2.
${ }^{d}$ See Ref. 1. 
the mapping from $x$ into $r$ is not sufficiently smooth, (that is, certain high derivatives of the map aren't bounded independent of $n$ ) the order of accuracy of the method will be reduced accordingly. Thus, the finite difference formula is fourth order on a smoothly varying mesh but only first order on a rougher mesh and, in fact, could become singular on a mesh having a local mesh ratio greater than 3.5 . When this is the case the parabolic method is preferred.

The Akima ${ }^{2}$ and Fritsch-Butland formulas are nonlinear algorithms for each $\dot{f}_{i}$. Consequently, the sum of the interpolants for data sets $(x, f)$ and $(x, g)$ is different from the interpolant for the sum of the data sets $(x, f+z)$. The other formulas do not have this defect in their initial approximations of $\dot{\mathrm{f}}_{i}$. However, after filtering according to (2.6), none of the interpolants are additive in the sense of (1.1) for $f=x+|x|, g=x-|x|$ when $x=0$ is a data point.

\section{B. Nonlocal Methods}

The most common nonlocal method for computing $\dot{\mathrm{f}}_{i}$ is the "not-a-knot" $c^{2}$ spline interpolation method, ${ }^{2}$ where $\dot{\mathrm{f}}_{i}$ is $0\left(\Delta \mathrm{x}^{3}\right)$ on an unequally spaced mesh or $O\left(\Delta x^{4}\right)$ away from the boundary on an equally spaced mesh. The $\dot{f}_{i}$ 's are calculated so the resulting interpolant has a continuous second derivative at the knots. As mentioned in Sec. II.A, the $\mathrm{C}^{2}$ spline interpolant will not necessarily satisfy Eq. (2.2) for monotone data. By allowing isolated discontinuities in the second derivative, a slightly deficient $\mathrm{C}^{1}$ monotone spline interpolant can be constructed in various ways. A possible solution is to filter the $C^{2}$ spline $\left\{\dot{\mathrm{f}}_{i}\right\}$ according to $\mathrm{Eq} .(2.6)$. This is the approach taken in the numerical examples presented here.

An algorithm that keeps the number of jumps in the second derivative small involves first computing $\left\{\dot{\mathrm{f}}_{i}\right\}$ for the complete spline interpolant in the 
interval $\left[x_{1}, x_{n}\right]$. If the interpolant is not locally monotone we locate point $x_{j}$ where $\dot{\mathbf{f}}_{j}$ is farthest outside the monotonicity region. We redefine $\dot{\mathbf{f}}_{j}$ according to Eq. (2.6) and solve for the complete spline interpolant in $\left[x_{1}, x_{j}\right]$ and $\left[x_{j}, x_{n}\right]$ using $f_{j}$ and $\dot{f}_{i}$ as boundary conditions. The resulting interpolant will have a break in the second derivative only at $x_{j}$. If none of the resulting $\left\{\dot{f}_{i}\right\}$ violate Eq. (2.6) we are finished. If some do violate Eq. (2.6), we repeat the process, break $\left[x_{1}, x_{j}\right]$ or $\left[x_{j}, x_{n}\right]$ into smaller subregions, and continue. This algorithm always will terminate if $\dot{f}_{1}$ and $\dot{\mathrm{f}}_{\mathrm{n}}$ are given at the boundaries and satisfy Eq. (2.6).

Another nonlocal approximation to $\dot{f}$ is the Fritsch-Carlson algorithm. 4 This method provides an approximation of $\dot{\mathrm{f}}$ that preserves piecewise monotonity in Eq. (2.1) with curves geometrically similar to those produced by the Fritsch-Butland method. Although we have not compared the Fritsch-Carlson method to the other methods in this report, we have included an example of specific data from their paper. ${ }^{4}$ From this example and other similar ones from their paper, we have found that the monotonicity-constrained algorithms using (2.3) or (2.6) perform similarly to the Fritsch-Carlṣon algorithm. The major difference is that the constraints (2.3) or (2.6) are much easier to implement. Also, the behavior of the (2.6) constrained interpolant at extrema in nonmonotone data sets is different. The Fritsch-Carlson algorithm clips the interpolant like the constraint (2.3) does.

\section{Boundaries}

At the boundaries we will use either the not-a-knot option for splines ${ }^{2}$ or an uncentered difference approximation. The second-order uncentered parabolic method used with the Akima, Fritsch-Butland, and parabolic 
algorithms is

$$
\dot{\mathrm{f}}_{i}=\frac{\left(2 \Delta \mathrm{x}_{i+1 / 2}+\Delta \mathrm{x}_{i+3 / 2}\right) \mathrm{s}_{i+1 / 2}-\Delta \mathrm{x}_{i+1 / 2} \mathrm{~s}_{i+3 / 2}}{\Delta \mathrm{x}_{i+1 / 2}+\Delta \mathrm{x}_{i+3 / 2}}
$$

or

$$
\dot{\mathbf{f}}_{i}=\frac{\left(2 \Delta \mathrm{x}_{i-1 / 2}+\Delta \mathrm{x}_{i-3 / 2}\right) \mathrm{s}_{i-1 / 2}-\Delta \mathrm{x}_{i-1 / 2} \mathrm{~s}_{i-3 / 2}}{\Delta \mathrm{x}_{i-1 / 2}+\Delta \mathrm{x}_{i-3 / 2}} .
$$

The third-order uncentered finite difference approximations used with the fourth-order interior formula are

$$
\begin{aligned}
& \dot{f}_{i}=\frac{-22 f_{i}+36 f_{i+1}-18 f_{i+2}+4 f_{i+3}}{-22 x_{i}+36 x_{i+1}-18 x_{i+2}+4 x_{i+3}}, \\
& \dot{f}_{i}=\frac{-2 f_{i-1}-3 f_{i}-6 f_{i+1}-f_{i+2}}{-2 x_{i-1}-3 x_{i}+6 x_{i+1}-x_{i+2}}, \\
& \dot{f}_{i}=\frac{22 f_{i}-36 f_{i-1}+18 f_{i-2}-4 f_{i-3}}{22 x_{i}-36 x_{i-1}+18 x_{i-2}-4 x_{i-3}},
\end{aligned}
$$

and

$$
\dot{f}_{i}=\frac{2 f_{i+1}+3 f_{i}-6 f_{i-1}+f_{i-2}}{2 x_{i+1}+3 x_{i}-6 x_{i-1}+x_{i-2}} \text {. }
$$

\section{NUMERICAL EXAMPLES}

The geometric and accuracy properties of the interpolants are compared on both. smoothly varying and rough data sets. When the derivatives are constrained by the extended de Boor-Swartz monotonicity limit Eq. (2.6), we call the resulting interpolant monotonically constrained (MC). 


\section{A. Monotone Example}

The Fritsch-Carlson RPN 15A data have been used to compare many different algorithms, $4,8,10$ some not included in this report. The data points are

\begin{tabular}{ll}
$\mathbf{x}$ & \multicolumn{1}{c}{$\mathrm{f}$} \\
7.99 & \\
8.09 & \\
8.19 & $2.76429 \mathrm{E}-5$ \\
8.7 & $4.37498 \mathrm{E}-2$ \\
9.2 & 0.169183 \\
10. & 0.469428 \\
12. & 0.943740 \\
15. & 0.998636 \\
20. & 0.999919 \\
& 0.999994
\end{tabular}

Figure 1 shows interpolation curves of the Akima, Fritsch-Butland, parabolic, fourth-order finite difference, complete spline, MC parabolic, MC fourth-order finite difference, and MC spline methods. These data show clearly that the Fritsch-Butland and MC methods are geometrically superior to the unconstrained methods. The simple constraint of Eq. (2.6) can convert an unacceptable geometric interpolant, such as the complete spline, into an excellent one. Note that the Akima algorithm, which was designed as a good geometric interpolant, fails to preserve monotonicity in this relatively simple example.

\section{B. Nonmonotone Example}

To interpolate the nonmonotone function $f(x)=e^{-x^{2}}, x \varepsilon[-1.7,1.9]$, the mesh was equally spaced with $\Delta x=3.6 /(n-1)$. This domain was chosen so the mesh points would not be symmetrical about the point of symmetry for the function.

Figure 2 shows that the higher order MC interpolants can be geometrically superior to the Akima, Fritsch-Butland, parabolic, and unconstrained interpolants when $n=5$. 


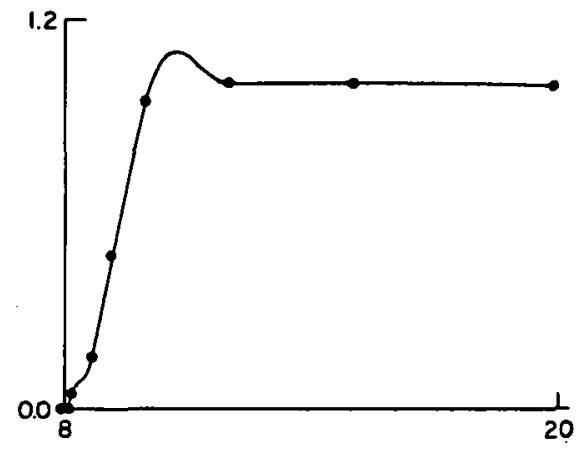

A. Akima

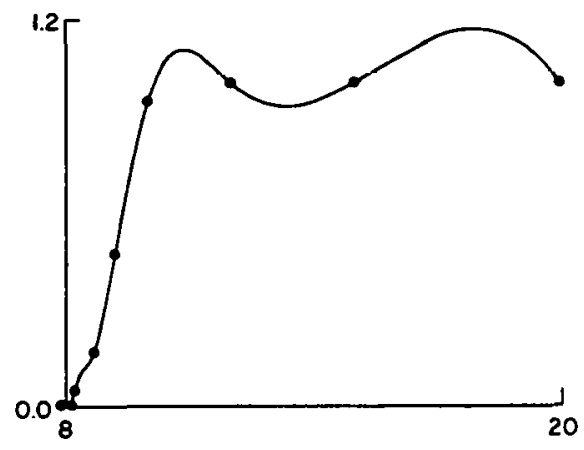

C. Complete spline

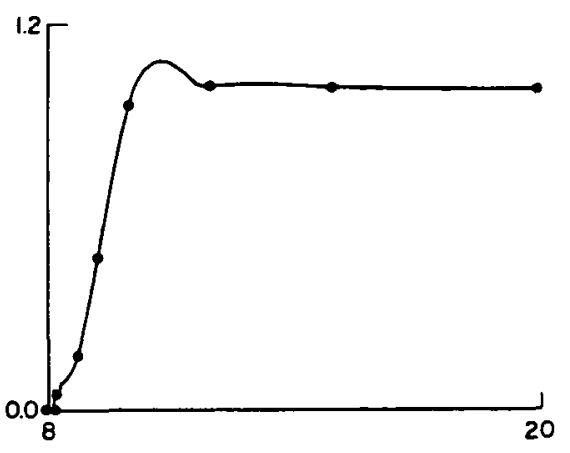

E. Parabolic

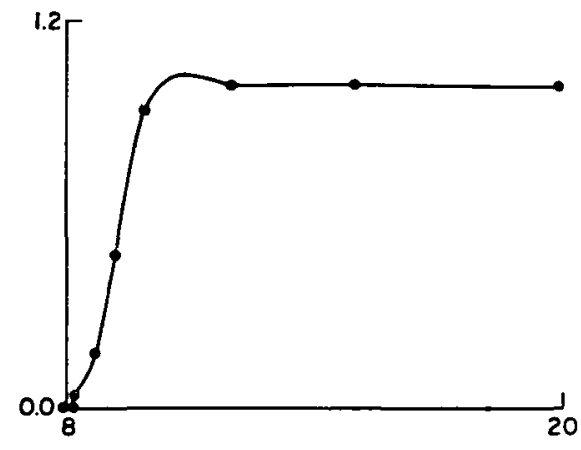

G. Finite difference

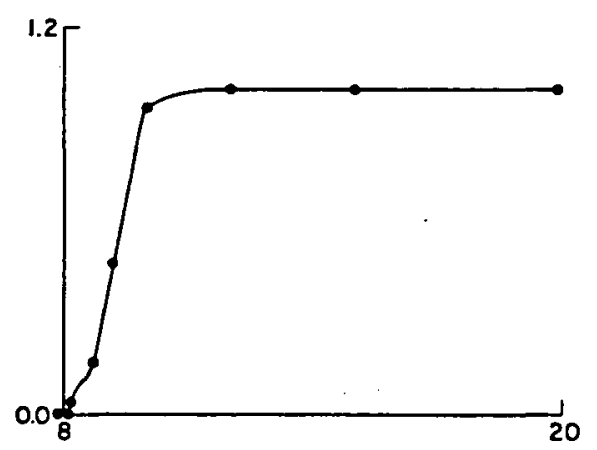

B. Fritsch-Butland

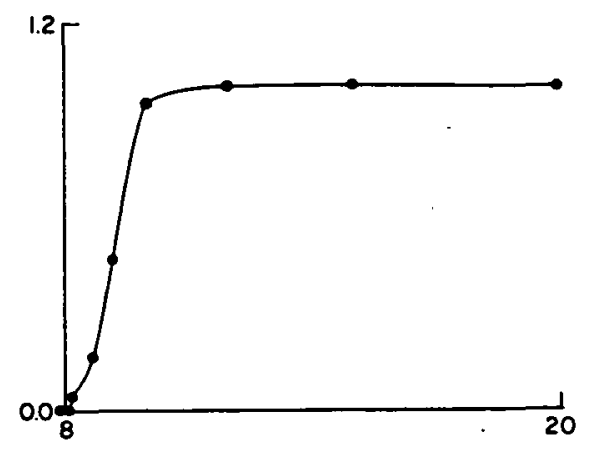

D. MC spline

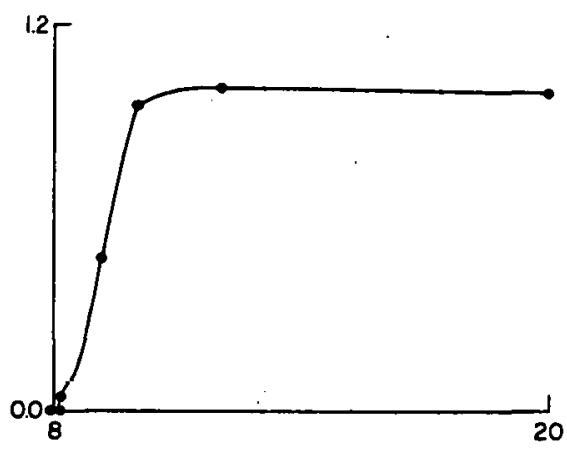

F. MC parabolic

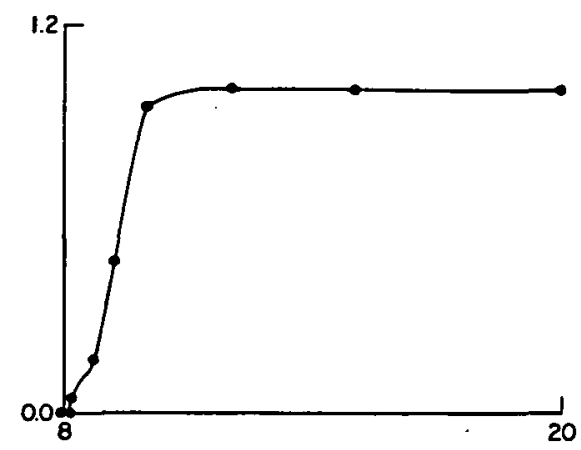

H. MC finite difference

Fig. 1. Interpolation curves for the RPN 15A data. 


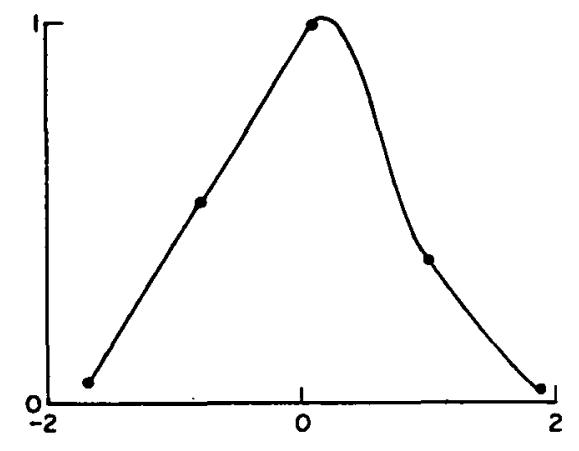

A. Akima

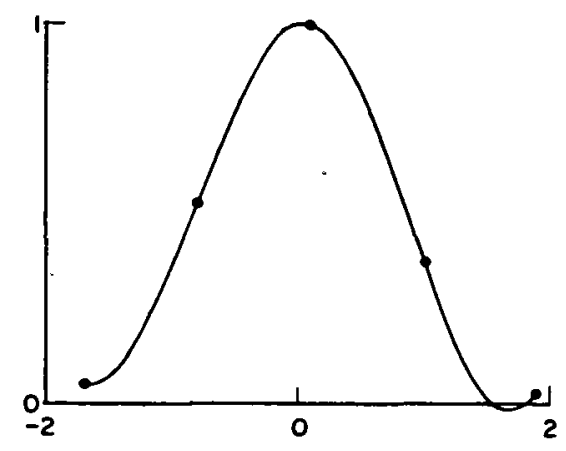

C. Complete spline

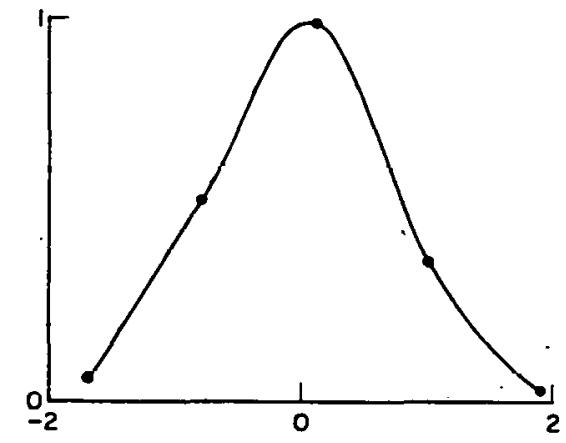

E. Parabolic

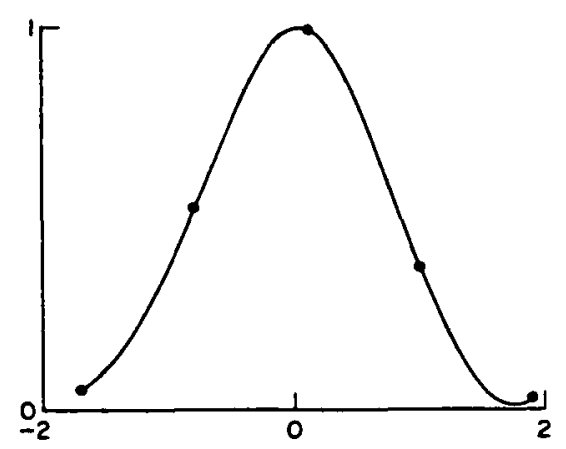

G. Finite difference

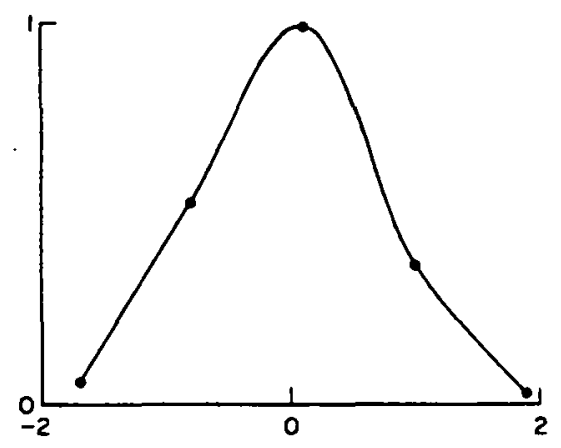

B. Fritsch-Butland

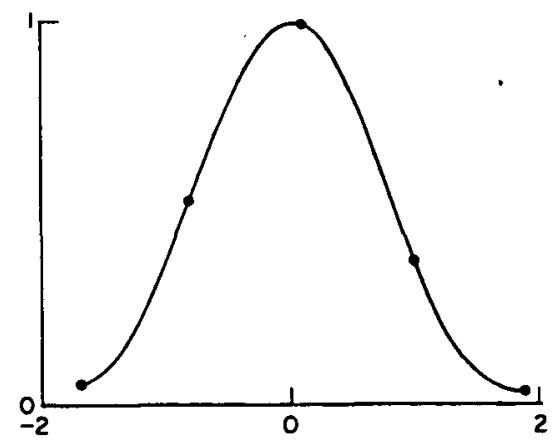

D. MC spline

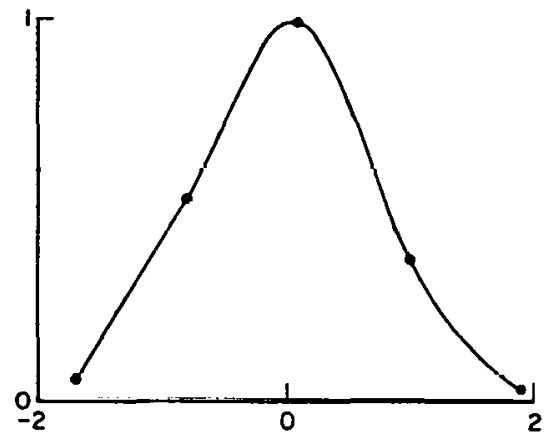

F. MC parabolic

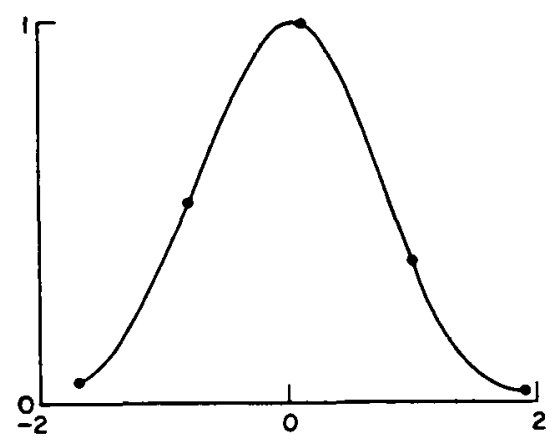

H. MC finite difference

Fig. 2. Interpolation curves for $f(x)=e^{-x^{2}}$. 
In Table II, the errors,

$$
\mathrm{L}_{2} \text { error }=\left(\int_{-1.7}^{1.9}\left[(\mathrm{Pf})(\mathrm{x})-\mathrm{e}^{-\mathrm{x}^{2}}\right]^{2}\right)^{\frac{1}{2}},
$$

of the interpolants are compared as the mesh is refined. Note that the higher order MC finite difference and spline methods are more accurate than the other methods both on the fine and coarse grids.

The MC methods agree with the corresponding unconstrained methods when $\mathrm{n}$ $=9$ and 33 but not when $n=17$ because the nonmonotonicity of the underlying function being interpolated.

TABLE I.I

COMPARISON OF THE METHODS FOR $f(x)=e^{-x^{2}}$

METHOD

METHUD
Akima
Fritsch-Butland
Parabolic method
Finite difference
Complete spline
MC parabolic
MC finite difference
MC spline

\section{Akima}

Fritsch-Butland

Parabolic method

Finite difference

Complete spline

MC parabolic

MC spline
$\mathrm{L}_{2}$ ERROR

$\underline{n=5} \quad \underline{n=9} \quad \underline{n=17} \quad \underline{n=33}$

6. $\mathrm{OE}-2$

4. $4 \mathrm{E}-2$

3. $9 \mathrm{E}-2$

2. $2 \mathrm{E}-2$

3. $5 \mathrm{E}-2$

3. $9 \mathrm{E}-2$

1. $5 \mathrm{E}-2$

1. 7E-2
6. $4 \mathrm{E}-3$

7. $3 \mathrm{E}-3$

4. $1 E-3$

3. $4 \mathrm{E}-3$

2. OE-3

4. $1 \mathrm{E}-3$

3. $4 \mathrm{E}-3$

2. $0 \mathrm{E}-3$
1. $\mathrm{OE}-3$

2. $4 \mathrm{E}-3$

4. $1 \mathrm{E}-5$

7. $4 \mathrm{E}-5$

4. OE-5

1. $9 E-3$

1. $9 \mathrm{E}-3$

1. $9 \mathrm{E}-3$
1. $3 \mathrm{E}-4$

1. $6 \mathrm{E}-4$

4. 3E-5

2. $3 \mathrm{E}-6$

1. $8 \mathrm{E}-6$

4. $3 F-5$

2. $3 E-6$

1. 8E-6

\section{SUMMARY AND CONCLUSIONS}

When only geometric considerations are important, any interpolant constrained to. stay within the de Boor-Swartz monotonicity limits using algorithm (2.6) is acceptable. The Fritsch-Butland does this automatically (that is, there are no conditional statements), but the approximating derivatives in other procedures must be filtered. 
When both geometric and accuracy considerations are important, the lower order methods (Akima, Fritsch-Butland, and parabolic) have larger truncation errors than the higher order constrained methods.

Therefore, we recommend first computing an approximation $\dot{\mathrm{f}}_{i}$ to $\frac{\mathrm{df}}{\mathrm{dx}}$ at the mesh points using either the local fourth-order finite difference method (Table I) or the nonlocal, but smoother, complete spline approximation. Before interpolating using Eq. (2.1), we filter $\left\{\dot{f}_{i}\right\}$ with Eq. (2.6) "so the interpolant will retain the important local monotonicity properties of the data. .

The simplicity of the filtering approach and the dramatic improvements in the interpolation curve far outweigh the cost of the extra few lines of code. Analyzing our numerical examples indicates that most cubic Hermite interpolation programs would be more versatile, robust, and often more accurate if a monotonicity constraint such as Eq. (2.6) were an option.

\section{ACKNOWLEDGEMENT}

I am grateful to Blair Swartz for providing me with much welcome advice in our many discussions during this work.

\section{REFERENCES}

1. G. I. Kerley, "Rational Function Method of Interpolation," Los Alamos National Laboratory report LA-6903-MS (1977).

2. C. de Boor, A Practical Guide to Splines, (Springer-Verlag, New York, Inc., New York, 1978).

3. C. de Boor and B. Swartz, "Piecewise Monotone Interpolation," J. Approximation Theory 21, 411-416 (1977).

4. F..N. Fritsch and R. E. Carlson, "Monotone Piecewise Cubic Interpolation," SIAM J. Numer. Ana1. 17, 2, 238-246 (1980).

5. D. F. McAllister, E. Pàssow, and J. A. Roulier, "Algorithms for Computing Shape-Preserving Spline Interpolations to Data," Math. Comp. 31, 717-725 (1977). 
6. J. M. Hyman, "Accurate Convexity-Preserving Cubic Interpolation," Los Alamos Scientific Laboratory informal report (November 1980).

7. J. Butland, "A Method of Interpolating Reasonable-Shaped Curves Through Any Data," Proceedings of Computer Graphics 80, (Online Publications Ltd., Northwood Hills, Middlesex, England, 1980), pp. 409-422.

8. F. N. Fritsch and J. Butland, "An Improved Monotone Piecewise Cubic Interpolation Algorithm," Lawrence Livermore National Laboratory preprint UCRL-85104 (1980).

9. J. M. Hyman, "The Numerical Solution of Time-Dependent PDEs on an Adaptive Mesh," LA-UR-80-3702 (1980) to be published.

10. J. M. Hyman, "Accurate Cubic Piecewise Monotone Interpolation," LA-UR-80-3700 (1980). 


\begin{tabular}{|c|c|c|c|c|c|c|c|c|c|c|c|}
\hline Page Range & $\begin{array}{c}\text { Domestic } \\
\text { Price }\end{array}$ & $\begin{array}{c}\text { NTIS } \\
\text { Price Code } \\
\end{array}$ & Page Range & $\begin{array}{c}\text { Domestic } \\
\text { Price }\end{array}$ & $\begin{array}{l}\text { ed in the Uni } \\
\text { A vails } \\
\text { nal Technica } \\
\text { US Departme } \\
5285 \text { Port } \\
\text { Springfiel } \\
\text { Microfiche } \\
\text { NTIS } \\
\text { Price Code }\end{array}$ & $\begin{array}{l}\text { States of Am } \\
\text { e from } \\
\text { nformation Se } \\
\text { of Commerce } \\
\text { oyal Road } \\
\text { VA } 22161 \\
3.50 \text { (A01) }\end{array}$ & $\begin{array}{c}\text { Domestic } \\
\text { Price }\end{array}$ & $\begin{array}{c}\text { NTIS } \\
\text { Price Code } \\
\end{array}$ & Page Range & $\begin{array}{c}\text { Domestic } \\
\text { Price }\end{array}$ & $\begin{array}{c}\text { NTIS } \\
\text { Price Code }\end{array}$ \\
\hline an1-025 & $\$ 5.00$ & $\mathrm{~A} 02$ & $151-175$ & $\$ 11.00$ & Aus & $301-325$ & $\$ 17.00$ & A14 & $451-475$ & $\$ 23.00$ & A20 \\
\hline $026-050$ & 6.00 & $\mathrm{~A} 03$ & $176-200$ & 12.00 & A09 & $326-350$ & 18.00 & A15 & $476-500$ & 24.00 & A21 \\
\hline $051-075$ & 7.00 & A04 & $201-225$ & 13.00 & A 10 & $351-375$ & 19.00 & A16 & $501-525$ & 25.00 & A22 \\
\hline $076-100$ & 8.00 & $\mathrm{~A} 05$ & $226-250$ & 14.00 & A11 & $376-400$ & 20.00 & A17 & $526-550$ & 26.00 & A23 \\
\hline $101-125$ & 9.00 & A06 & $251-275$ & 15.00 & A12 & $401-425$ & 21.00 & A 18 & $551-575$ & 27.00 & A24 \\
\hline $126-150$ & 10.00 & A07 & $276-300$ & 16.00 & A 13 & $426-450$ & 22.00 & A 19 & $\begin{array}{c}576-600 \\
601 \text {-up }\end{array}$ & $\begin{array}{c}28.00 \\
\dagger\end{array}$ & $\begin{array}{l}\text { A25 } \\
\text { A99 }\end{array}$ \\
\hline
\end{tabular}

†Add $\$ 1.00$ for each additional 25 -page increment or portion thereof from 601 pages up. 


\section{Los Alaños}

